\title{
Carnets
}

Revue électronique d'études françaises de l'APEF

Première Série - 1 Numéro Spécial | 2009

Cultures littéraires : nouvelles performances \& développement

\section{La série télévisée "Kaamelott" ou la matière arthurienne revisitée}

Marie-Manuelle da Silva

\section{(2) OpenEdition \\ Journals}

Édition électronique

URL : http://journals.openedition.org/carnets/4064

DOI : $10.4000 /$ carnets.4064

ISSN : 1646-7698

Éditeur

APEF

Édition imprimée

Date de publication : 1 juin 2009

Pagination : 307-318

Référence électronique

Marie-Manuelle da Silva, "La série télévisée "Kaamelott" ou la matière arthurienne revisitée », Carnets

[En ligne], Première Série - 1 Numéro Spécial | 2009, mis en ligne le 16 juin 2018, consulté le 01 mai 2019. URL : http://journals.openedition.org/carnets/4064 ; DOI : 10.4000/carnets.4064

\section{(c) (i) ()ㅜㄹ}

Carnets est mis à disposition selon les termes de la licence Creative Commons - Atribution - Pas d'utilisation commerciale 4.0 International. 


\title{
LA SÉRIE TELEVISÉE "KAAMELOTT" \\ ou la matière arthurienne revisitée
}

\author{
MARIE-MANUELLE DA SILVA \\ Universidade do Minho \\ mmcsilva@ilch.uminho.pt
}

\begin{abstract}
Résumé
La légende du roi Arthur a profondément marqué la culture occidentale et semble aujourd'hui revenir en force, si on en juge par les nombreux avatars comiques ou pseudo-épiques qui surgissent sur les supports médiatiques les plus variés. En effet, la Matière arthurienne a connu de nombreuses réécritures depuis le Moyen Âge en passant par l'Angleterre victorienne (qui en orchestre la "renaissance"), jusqu'à l'époque contemporaine, séduite par les personnages et les aventures du royaume d'Arthur. Nous proposons d'aborder ces questions à la lumière de la série télévisée française "Kaamelott" de Alexandre Astier et Yves Robin créée en 2006, série dite "de fantaisie historique" qui s'inspire de la Matière arthurienne et qui connaît une popularité croissante, montrant que les inspirations médiévales et «celtiques» sont aujourd'hui très prégnantes.
\end{abstract}

\begin{abstract}
The legend of King Arthur deeply influenced Western culture and seems more than ever in vogue today, judging by numerous comic adaptations and mixed-media 'pseudo-epics'. Indeed, the legend has undergone numerous re-interpretations since the Middle Ages, notably in Victorian England (which gave rise to its 'renaissance') and in the contemporary era, which has been fascinated with the characters and adventures of Arthur's kingdom. These questions will be addressed in the light of the French television series "Kaamelott" by Alexandre Astier and Yves Robin, which was created in 2006. Described as a "historical fantasy" inspired by the Arthurian legend, the series has become increasingly popular, indicating how medieval and celtic themes carry particular meaning today.
\end{abstract}

Mots-clés: Adaptation, Réécriture, Série télévisée, Matière arthurienne

Keywords: Adaptation, Reinterpretation, Television series, Arthurian theme 
Le phénomène relativement récent de la multiplication des adaptations de textes littéraires (au théâtre, au cinéma, à la télévision et dans la BD) est la conséquence de changements dans les champs artistiques et culturels. L'apparition d'arts narratifs propres aux nouveaux canaux de diffusion favorisent la prolifération des récits et conduisent à situer la littérature comme un discours parmi d'autres discours avec lesquels elle entretient plusieurs modes d'interaction, de conversion et de contamination.

La légende du roi Arthur a profondément marqué la culture occidentale et semble aujourd'hui revenir en force, si on en juge par les nombreux avatars qui surgissent sur les supports médiatiques les plus variés. L'un de ces nouveaux textes arthuriens, la série télévisée française "Kaamelott" de Alexandre Astier et Yves Robin créée en 2006, a attiré notre attention en sa qualité de phénomène télévisuel populaire s'inscrivant dans l'histoire culturelle et puisant dans le réservoir des variantes successives dès les origines du roman arthurien.

Nous expliciterons dans un premier temps le contexte de diffusion de la série et nous aborderons des problématiques liées à sa spécificité en tant que média pour mieux appréhender la singularité de l'actualisation du mythe du roi Arthur dans Kaamelott. Nous tenterons ensuite de situer la série analysée dans la stratification intertextuelle des réécritures contemporaines de la matière Arthurienne en tentant d'en définir les coordonnées entre le pôle de la répétition et de la familiarité basée sur la tradition attestée des réécritures du Moyen Age et celui de la nouveauté et de la singularité, (dé)jouant avec les contraintes propres aux caractéristiques génériques et structurelles de la "matière" télévisuelle prise dans ses propres mythes et ses dynamiques commerciales qui affectent en retour les produits qu'elle charrie.

Cette communication pose les premiers jalons d'une réflexion qui devra être approfondie et dont les hypothèses devront être confirmées quand la série aura été totalement diffusée (la dernière saison le sera en 2009).

\section{La télévision entre réalité et fiction}

Si la télévision ne paraît pas morte en tant que media (Missika, 2006) comme on l'a parfois annoncé, son statut et son usage se sont considérablement transformés. Dans les années 80, Umberto Eco distinguait la néo-télévision de la paléo-télévision en ces termes: 'La caractéristique de la Néo-TV, c'est le fait qu'elle parle de moins en moins du monde extérieur (ce que la Paléo-TV faisait ou feignait de faire). Elle parle d'elle-même et du contact qu'elle est en train d'établir avec son public." (Eco, 1985: 198). U. Eco définissait ainsi un nouveau modèle de communication télévisuelle, (la néo-télévision), dont la téléréalité serait l'apothéose, comme le démontre Dominique Mehl en 2002 dans les Cahiers internationaux 
de sociologie (Mehl, 2002). L'auteur, reprenant les concepts de U. Eco, souligne que, dans le cas de la néo-télévision, le pouvoir acquis par le public est à l'origine d'un "déplacement du centre de gravité des programmes de la crédibilité des énoncés vers la crédibilité de l'énonciation elle-même" provoquant "la neutralisation de la dichotomie entre information et fiction, les programmes mélangeant les deux." (idem: 90). La Paléo-TV, une fenêtre ouverte sur le monde, a laissé place à la Néo-TV indépendante pour qui 'l'écran devient enfin comme la vraie vie" (ibidem: 91).

Au début des années 90, Francesco Casetti et Roger Odin, reprenant le binôme de Umberto Eco, présentaient la paléo-télévision comme une institution fondée sur un projet d'éducation culturelle et populaire vouée à la transmission de savoirs, la néo-télévision étant, quant à elle, la mise en scène d'un processus d'interactivité avec le public. La transformation d'un "espace de formation" en "un espace de convivialité" a conduit à ce que l'espace de la réalisation et celui de la réception coexistent au sein d'un même espace télévisuel se confondant avec l'espace quotidien (Casetti et Odin, 1990).

A la même époque, Pierre Chambat et Alain Ehrenberg mettaient l'accent sur les causes de ces transformations: "concurrence accrue entre les chaînes, épuisement des variétés classiques, contraintes de coûts alors que la durée d'émission s'est considérablement accrue, fidélisation d'un public devenu volage, souci de gagner une audience plus jeune, renouvellement des formes télévisuelles par injection d'interactivité." (Chambat, Ehrenberg, 1993: 5). Aujourd'hui, de l'ordinateur au téléphone portable, les écrans se sont multipliés, la consommation de programmes s'émancipe des diffuseurs (visionnements en différé sur l'internet, piratage, téléchargement), les offres de vidéo à la demande (VOD) remportent un succès croissant, internet accueille de plus en plus de chaînes (voir la chaine NoLife de culture "geek"1), les nouveaux canaux de diffusion ont considérablement diversifié l'offre avec la télévision numérique terrestre (TNT) dont les contenus thématiques spécifiques ôtent aux chaînes généralistes privées comme publiques une part croissante de l'audience. Proposer un rendez-vous régulier avec le public alors que les grilles de programmes sont virtuellement maîtrisées par le spectateur et non plus par le diffuseur pose de nouvelles contraintes, alors même que la télévision doit se redéfinir pour trouver sa place au sein de la vie culturelle aux cotés d'autres œuvres de création comme le cinéma, le spectacle vivant, le livre etc.

Même si le spectateur n'est plus astreint à la linéarité du visionnement unique selon des coordonnées spatio-temporelles imposées, le média télévision n'est pas mort puisque

\footnotetext{
${ }^{1}$ Le terme geek (sous-genre du freak, monstre de foire anglo-saxon) est employé pour désigner le technophile génie de l'informatique et par extension son univers culturel proche de l'univers fantastique et de science fiction (jeux de rôle, jeux vidéos, manga etc.).
} 
qu'il "secrète" encore des genres qui lui sont propres et qui montrent sa vitalité ${ }^{2}$ La série télévisée en est un exemple: réalisée en fonction des spécificités du format du média, elle n'existe que par celui-ci, à la différence des films de cinéma qui peuvent être diffusés à la télévision mais dont les images sont tronquées pour le petit écran. L'ancrage pragmatique de la série, son lien au temps quotidien et historique, est également de nature exclusivement télévisuelle, car seul le cadre domestique de la télévision permet un rapport régulier, ou du moins répété et familier avec l'univers des séries. Ainsi, la série télévisée est un objet culturel articulé au média qui ne se réduit pas à son support de diffusion ou de médiation.

Le cadre domestique est maintenant concurrencé par d'autres modes de diffusion et l'effritement entre réalité et fiction propre à la néo télévision est reconfiguré dans des séries qui créent leur propre rapport au monde à travers des modes particuliers de relation entre réalité et fiction sur lesquels nous reviendrons. Le glissement entre la "mission" de transmission du savoir propre à une certaine conception (mythique ?) du service public vers la télévision comme espace de convivialité est lui aussi marqué par une évolution. De nouvelles conditions de lecture ont été rendues possibles par les supports enregistrés, les contenus peuvent être examinés, discutés et analysés par le public dont les interventions oscillent entre discours critique et invention fictionnelle: aux côtés de commentaires sur les films ou les séries, les intervenants élaborent des conjectures et proposent même des extensions hypothétiques aux fictions. En effet, internet regorge de sites où les fans mettent à l'épreuve leur cohérence et dénoncent leurs contradictions, comme le montre Richard Saint-Gelais en observant les discours produits à propos de la série Twin Peaks de David Lynch (1990) et du film Blade Runner de Ridley Scott (1982) (Saint Gelais, 1999). Ces nouvelles pratiques ont affecté en retour les fictions télévisuelles dont l'écriture et le format portent les marques.

\section{La série Kaamelott: un objet pris dans le media télévision, entre réalité et fiction}

\section{Contexte de diffusion: l'influence d'un genre.}

Kaamelott apparaît dans un paysage audiovisuel en pleine mutation, marqué par l'évolution du statut et de l'usage de la télévision. Des transformations ont également affecté le genre télévisuel que constitue la série et le modèle américain a largement influencé la production nationale.

\footnotetext{
${ }^{2}$ Voir GLEVAREC, Hervé (2007). "La télévision est enfin un média : discussion à propos de La fin de la télévision de Jean-Louis Missika", document non publié, IFRESI-CLERSE. <URL: www.univlille1.fr/clerse/site_clerse/pages/accueil/fiches/Glevarec.htm>.
} 
Comme le rappelle Martin Winckler dans un article publié dans le Monde Diplomatique du mois de juillet 2006, les séries américaines perçues comme "vulgaires, sans imagination et politiquement correctes" au début des années 2000, sont devenus aujourd'hui, pour beaucoup d'entre elles, des objets culturels cultes. Selon l'auteur, le spectateur "dénué de préjugés", a pu se rendre compte qu'au-delà du divertissement, les séries - des plus réalistes aux plus débridées - abordaient tous les genres et témoignaient d'un certain engagement aussi bien social que politique. Les séries américaines ont réconcilié le monde et la fiction, en créant un niveau de réalité singulier où les fictions s'ancrent dans des univers diégétiques vraisemblables comme les hôpitaux ou les services de police spécialistes en criminologie et autres cellules anti-terroristes ${ }^{3}$, participant d'une certaine manière à l'effritement entre fiction et réalité annoncé par Umberto Eco.

\section{Les séries françaises: quelles fictions pour la nouvelle réalité de la TV ?}

Quant aux séries françaises, les déclarations en leur faveur se multiplient ${ }^{4}$ et ce que l'on nomme désormais "la nouvelle fiction nationale" 5 cohabite, toutes chaînes confondues, avec les séries américaines à succès. On pourrait établir une polarité entre la chaîne privée Canal Plus, qui diffuse des séries d'auteur ${ }^{6}$ sans pour autant atteindre les taux d'audience escomptés ; et la chaîne M6, dont une grande partie de la programmation est depuis toujours consacrée aux séries américaines et qui accueille les séries les plus populaires comme Kaamelott, pierre angulaire de sa programmation (ou encore la comédie Les Bleus dont le cadre est un commissariat et Off Prime qui joue sur la téléréalité avec des "people" jouant leur propre rôle).

II est aisé de constater que les remarques émises par Pierre Chambat et Alain Ehrenberg en 1993 sont toujours vraies aujourd'hui: l'évolution de la fiction (influencée par les modèles américains, notamment par la chaîne HBO) a favorisé un type d'écoute particulier. Les séries sont construites pour être suivies et comprises dans la relation d'écoute flottante avec le récepteur, permettant aux téléspectateurs de "gérer" l'abondance télévisuelle. Nous ne pouvons nous attarder ici sur les nombreuses modalités adoptées dans les séries les plus récentes ${ }^{7}$ mais notons que leur durée moyenne à tendance à s'écourter (le format du feuilleton traditionnel est de $90 \mathrm{mn}$ alors que la plupart des séries font $52 \mathrm{mn}$ ou

\footnotetext{
${ }^{3}$ On pense à E.R/Urgences ; Dr.House ou Nip/tuck dans des styles complètement différents ; CSI ou Numbers ou encore $24 h$ chrono.

4 '"ll faut inventer une nouvelle fiction française, la sortir de l'ornière. Elle doit être plus imaginative sur la forme, plus proche du téléspectateur.", dit Laurent Storch, directeur des programmes de TF 1 "TF1 fait du neuf avec beaucoup de vieux" » Christophe Joly. In Journal 20mn.fr du 11/09/08 consulté le 11/09/2008.

${ }^{5}$ Dont elle s'inspire parfois : voir RIS, qui rappelle CSI ou encore l'Hôpital, Grey's Anatomy.

6 La Commune (série en huit épisodes de Abdel Raouf Dafri) ; La Nouvelle Trilogie (série sur le cinéma X de Bruno Gaccio) ou Scalp (de Xavier Durringer).

${ }^{7}$ Voir entre autres : L’Année des Séries 2008 (2008) Winckler M., Boutet M. (dir.). Editions (Hors Collection).
} 
$40 \mathrm{mn}$ pour les séries américaines) et leur structure narrative, qu'elles soient unitaires ou à suivre, se complexifie ${ }^{8}$.

\section{Genèse de Kaamelott}

Obéissant à des dynamiques commerciales, le format de Kaamelott a subi des transformations qui ne sont pas sans relation avec son succès populaire ${ }^{9}$.

Inspirée d'un court métrage de quatorze minutes Dies iræ ${ }^{10}$, la série créée en 2005 par Alexandre Astier et Jean-Yves Robin (membre de la société CALT qui la produit) est ancrée dans la Grande-Bretagne médiévale, sur laquelle règne un Roi Arthur progressiste et tolérant en son château de Kaamelott. Entouré par une belle famille querelleuse, marié à une Guenièvre stupide et naïve, affublé de Chevaliers de la Table ronde couards et imbéciles ou archaïques, sanguinaires et même traîtres, l'Arthur de la série peine à mener à bien sa quête du Graal.

La série a d'abord été programmée sous la forme d'épisodes d'environ $3 \mathrm{mn}$ (saisons ou Livre I à IV) puis de $7 \mathrm{mn}$ (saison V) et aura un format de 40mn pour les 9 épisodes de la saison VI qui sera diffusée au printemps 2009 en première partie de soirée et par blocs de 3 épisodes ${ }^{11}$.

Son succès commercial - c'est l'un des programmes les plus rentables des quatre principales chaînes françaises - explique ces transformations: le passage de la shortcom ou comédie à gags conçue pour être vue occasionnellement (parce qu'elle constitue une unité narrative), à une modalité plus longue à la narration plus complexe témoigne de l'évolution du statut de Kaamelott ${ }^{12}$. Le succès de la série et la fidélité de son public ont changé les enjeux de son écriture: il ne s'agit plus seulement de séduire pour capter et fidéliser un public, mais aussi de répondre à ses attentes que les nouvelles pratiques de lecture viennent conditionner. En effet, le téléspectateur n'est plus astreint à la linéarité d'un visionnement unique puisque certains épisodes sont disponibles sur internet ou en DVD. Un important réseau s'est construit à partir et autour de Kaamelott, où les fans échangent leurs opinions, traquent les contradictions ou les incohérences et proposent leur analyse sur l'évolution de la

\footnotetext{
${ }^{8}$ Voir Mittell, J. (2006) Narrative Complexity in Contemporary American Television. The Velvet Light Trap Number 58, Fall 2006, pp. 29-40.

${ }^{9}$ La série est diffusée depuis janvier 2005 sur M6 et s'est exportée : depuis 2006 en Suisse (sur TSR 2) et en Belgique (Club RTL) et depuis 2007 au Québec, avec des adaptations (dits “ dérivés ») en Italie et en Espagne). ${ }_{10}$ Dont l'action se situe à l'époque arthurienne- réalisé et produit en 2003 Alexandre Astier et pour lequel il a remporté le prix du Public du festival Off-Courts en 2003 et le prix du festival Comédia Juste pour rire de Montréal 2004.

${ }_{11}$ C'est le mode de diffusion de la plupart des séries actuellement programmées à la télévision.

12 Kaamelott a été l'objet de deux programmations spéciales en « prime time » de deux fois cinquante-deux minutes en 2007 reprenant les épisodes quotidiens et quelques inédits.
} 
série dans des forums ou en contribuant aux encyclopédies interactives en ligne comme Wikipédia $^{13}$.

On peut supposer que ces dynamiques affectent en retour l'écriture de Kaamelott et le Livre II initie un mouvement chronologique inédit en établissant un lien entre les Livres I et III dans lequel les références aux autres livres se multiplieront. L'intrigue principale, que les scénaristes TV nomment arc, sera poursuivie en rhizome dans les Livres suivants: un arc central à suivre d'un livre à l'autre sur lequel se greffent des arcs mineurs constituent une structure diégétique adaptée à des pratiques scripturales et lecturales qui s'auto influencent.

La série s'inaugure avec des sketches indépendants sur un arrière fond commun: l'île de Bretagne du Ve siècle après Jésus-Christ pendant l'effondrement de l'Empire, quand le christianisme naissant affronte les anciennes traditions celtes. Quand la société progressiste que le roi Arthur de Kaamelott essaie de développer est sur le point de se désagréger, la structure narrative tend à se ramifier et à se complexifier en s'engageant dans «la voie mythique ». Les dieux, las des affronts répétés d'Arthur - l'adultère avec la femme d'un chevalier, le peu d'entrain pour la quête du Graal... - décident de lui envoyer un signe inquiétant sous la forme d'un mystérieux personnage nommé Méléagant qui a pour dessein la mort du roi et l'avènement de Lancelot. Arthur, poussé au suicide, tente de mettre fin à ses jours au moment même où Lancelot vient pour le tuer, à la fin du Livre V. Dans le Livre VI Arthur va se remémorer ses racines et son histoire, de son adolescence à Rome à sa fédération des clans bretons.

\section{Kamelott et la matière arthurienne}

Kaamelott d'Alexandre Astier a repris l'un des plus grands mythes de l'occident à la suite de nombreux auteurs qui en ont assuré l'omniprésence jusqu'à l'époque contemporaine. La prégnance de l'imaginaire arthurien semble renvoyer à ce que Anne Besson nomme "une médiévité fantasmée ne cessant de ressurgir dans l'histoire culturelle, comme si son reflet demeurait suffisamment flou pour que l'ont ait pu y projeter les idéologies et les esthétiques les plus diverses." (Besson, 2007: 6).

Par ailleurs, comme l'auteure le souligne, la diversité du corpus arthurien est rendue possible par la fécondité d'un canevas narratif et d'une galerie de personnages aptes à se prêter aux variations qui se nourrissent, à leur tour, les unes des autres dans un processus "qui d'une fiction fait un mythe" (idem). Mythe moderne qui suscite la fascination collective en se posant comme fondateur et exemplaire (Lévi-Strauss) ; mythe littéraire qui s'impose en

\footnotetext{
${ }^{13}$ II n'y a, à notre connaissance, que peu d'études de spécialistes sur la série : les deux livres de vulgarisation de Eric Le Nabour préfacés par Martin Aurell (Le Nabour, Eric (2007). Kaamelott T1 et T2. Éditions Perrin.
} 
dehors de toute origine assignable; mythe ethnographique aux archétypes primitifs récurrents, Arthur est à la fois "un héros fixé par l'histoire et libéré par la fiction" (idem: 7).

Selon Anne Besson, le mythe littéraire d'Arthur correspond à l'approche lévistraussienne du mythe dont la dualité permet constance et évolution en dépassant les antinomies par la création fictionnelle. La somme des représentations du mythe littéraire n'en actualisent que le nécessaire à chaque avatar et matérialisent l'éternel retour du temps mythique. Si Arthur est à l'origine d'une production narrative importante et pérenne, c'est parce qu'il concentre des systèmes d'opposition qui balisent l'imaginaire entre familiarité et nouveauté. La Matière de Bretagne très ancienne dans le champ de la fiction s'est renouvelée au long de son histoire permettant 'la reconnaissance d'un corpus, d'une œuvre collective cohérente, quand l'obscurité - la variante, la contradiction - figurait la part de liberté créatrice, l'espace des initiatives individuelles dans la formulation des hypothèses." (Besson, 2006: 175).

La forme incarnée du mythe donnée par Alexandre Astier s'inscrit dans ce mouvement de "répétition/variation, statisme/renouveau" (idem). Elle intègre les pôles de l'histoire et du mythe entrainant des codes socio sémiotiques entre lesquels la fiction oscille: ceux de la tradition littéraire du corpus arthurien d'emblée polarisée entre prétention à (re)dire la vérité et les variantes divergentes balisées par la résistance de l'archétype, et ceux de la transfictionnalité que Saint-Gelais définit comme le franchissement des frontières censées séparer différentes œuvres par des personnages ou des univers fictifs (idem: 247). Ces fusions typiques des avatars les plus récents du corpus pourraient être interprétées comme une mise en abyme des débats sur la télévision en reprenant le jeu des oppositions réalité/ fiction, espace de formation/espace de convivialité, culture/divertissement, qualité/audimat.

\section{L'histoire et le corpus médiéval}

S'inspirant du genre populaire de la "fantasy arthurienne" et des plus nobles littératures de genre où se confondent souvent l'existence historique d'Arthur et l'histoire littéraire des récits le concernant, Kaamelott opère une fusion entre des codes génériques devenus conciliables par le jeu de l'intertextualité dans le corpus arthurien lui-même.

Le monde dans lequel évoluent les personnages de la série circulent en permanence entre deux époques séparées par six ou sept siècles d'histoire, confusion également présente dans les récits qui se référent à Arthur de Bretagne (L'histoire des rois de Bretagne 
de Geoffroi de Monmouth en $1138^{14}$, Robert Wace qui le "traduit" ou, plus tard, Chrétien de Troyes qui "invente" les chevaliers de la table ronde). Si Arthur a bien vécu au Vle siècle, il nous est surtout familier par des textes des XIle et XIIle siècles et il semble difficile d'évoquer l'Arthur historique sans faire référence au héros de la littérature et du Cycle du Graal.

Les deux mondes qui coexistent dans la série (les personnalités d'Arthur qui y sont associées) renvoient d'une part à la période violente de la mise en place des royaumes "barbares" sur les ruines de l'empire romain et d'autre part au Moyen Age flamboyant des Xlle et XIIle siècles comme les châteaux, les vêtements, les armures et les scènes d'amour courtois le laissent présumer. On peut d'ailleurs émettre l'hypothèse que le clivage entre la civilisation romaine représentant une certaine idée de la cité, de la culture, des arts et des lettres et les barbares de culture essentiellement rurale et orale trouve une résonance dans la situation géopolitique actuelle (mondialisation/ occidentalisation /impérialisme), mais qu'il exprime aussi la tension entre le canon culturel (et cultivé) et les médiacultures que Maigret et Macé disent "constituées des produits issus des industries culturelles ${ }^{15}$, et destinées à un grand public hétérogène et non à une masse populaire homogène comme le terme 'médias de masse' parfois utilisé, pourrait le faire penser." (Maigret et Macé, 2005: 11).

\section{Les autres références: la transfictionnalité}

Si Alexandre Astier a construit sa "géographie Arthurienne sur un héritage tellurique primordial" (Besson, 2006: 8), il additionne à son texte des références plus contemporaines, références elles-mêmes issues des stratifications que Anne Besson définit comme "évolution intervolumiques au jeu du familier et de l'inventé intertextuels" (idem: 14). A la complexité des versions délimitant le corpus médiéval viennent s'ajouter des références hétérogènes formant une hybridation générique entre historique et merveilleux. Dans Kaamelott, les croyances des "barbares" (vénération des forces naturelles, expression de divinités multiples, génies, fées etc.) sont actualisée par le biais des références à l'univers de la fantasy (High fantasy), notamment les jeux de rôle et le "porte-monstre-trésor" (dungeon crawling) typique de l'univers du jeu médiéval Donjons et dragons. Ces jeux (de plateau, grandeur nature ou vidéo) permettent de choisir et d'incarner des personnages qui, selon

\footnotetext{
${ }^{14}$ Qui hisse Arthur au rang de héros politique national, il déplace la cour d'Arthur et ses enjeux dans le contexte du Xlle. En extirpant Arthur du temps, Monmouth rapproche l'histoire du public et actualise ainsi les préoccupations dont elle pourrait être le vecteur tout en la suspendant dans le temps mythique.

${ }^{15}$ Le concept d'industries culturelles, apparu au XIXème siècle, s'est développé au XXème siècle pendant la révolution industrielle et la mutation des sociétés industrielles vers des sociétés post-industrielles.
} 
leurs attributs, disposent de pouvoirs et d'alliances pour vivre des aventures scénarisées par un maitre de jeu ${ }^{16}$.

On constate d'abord une référence directe au film Sacré Graal ! des Monty Python (1974) dans l'épisode Un bruit dans la nuit (DVD Livre I, T2 ํ11), dans lequel Bohort est terrifié à l'idée de rencontrer un "lapin adulte" dans la forêt (une allusion au film des Monthy Python où Bors est le premier chevalier victime du 'lapin vorpal'). Cette parodie des Monthy Python peut aussi se lire comme un discours sur l'insuffisance du cinéma à montrer le mythe arthurien qui devient un objet cinématographique inaccessible: comment atteindre ce que l'on ignore (le Moyen Age) et ce qui échappe (la dimension mythique) ? Alexandre Astier opte lui aussi pour le décalage que permet la parodie, principalement basée sur l'anachronisme. Cette "recette" exploitée dans les comédies populaires comme Les Visiteurs (de Jean-Marie Poiré) est ici doublée par l'allusion au cinéma d'auteur: Arthur est en avance sur son temps qu'il regarde avec la même distance qui sépare le cinéma du mythe arthurien dans le film des Monthy Python. Le langage des personnages composé d'argot (comme dans les dialogues des films cultes de Michel Audiard) et d'expressions plus contemporaines renforcent ce "désajustement" qui permet de mettre en valeur les mœurs de la France et de l'Occident mais aussi d'amuser par des situations cocasses au premier degré.

A mesure que Kaamelott s'engage dans la voie des temps obscurs, à partir du Livre $\mathrm{V}$, les références au film Excalibur de John Boorman (1981) deviennent explicites (dès les premiers livres, le décorum de la Table Ronde et les trompettes dans le générique y faisaient déjà référence), le quotidien profane s'effaçant au profit de la dimension mythique. Dans le film, une géographie légendaire, des décors et des costumes exempts de pseudo réalisme historique (ils sont brillants et artificiels) et quelques motifs médiévaux constituent une ambiance plus qu'une idéologie précise. La cohérence de Excalibur est d'ordre mythique, on ne recherche pas l'identification par le biais de ce qui est humain, compréhensible et transposable dans une situation vécue, mais au contraire le dépaysement par le mythe, une autre façon de vivre son rapport au monde.

\section{Conclusion}

La série Kaamelott compose avec les sources médiévales attestées mais aussi avec les réécritures de ses prédécesseurs directs au moyen de références à la fantasy (pour le maniement du merveilleux), ou à des films d'auteurs qui choisissent de représenter le mythe arthurien d'une manière singulière en réagissant aux représentations du Moyen Age au

\footnotetext{
${ }^{16}$ Voir DVD du Livre I : Le Labyrinthe, (nำ34); La Grotte de Padraig (nำ14); Le Chaudron rutilant (nํ36).
} 
cinéma. Astier semble assumer que toute représentation du Moyen Age est une spéculation (pour ne pas dire une imposture ou de la camelote - marchandise de pacotille - comme le nom de la série le suggère) et il confère une valeur similaire aux travaux des historiens et à ceux des écrivains, cinéastes ou autres créateurs.

II apparaît cependant que le réalisateur de la série cherche un équilibre dans une autre polarité qui fait écho à celle de la critique Arthurienne: d'un coté les références à l'histoire culturelle et à ses formes canoniques (qu'elles proviennent de la "haute" ou de la "basse" culture) et de l'autre les emprunts aux produits de l'industrie culturelle, reflets d'une tension entre la création dite d'auteur et les compromis du phénomène commercial que les "produits dérivés" autour de Kaamelott prennent en relais (DVD, bande dessinées mais aussi livres signés par des spécialistes du Moyen Age, même si on peut parler d'ouvrages de vulgarisation).

Kaamelott questionne la "mission" de transmission du savoir propre à une certaine conception du service public et pose la TV comme espace de convivialité à appréhender à travers plusieurs niveaux de lecture tissés par des références qui fonctionnent en réseau et rattachent différentes "communautés" de téléspectateurs. II semblerait que dans la société de flux qui est la nôtre l'espace social

se donne comme matière inorganisée à canaliser, comme pelote intriquée à démêler, comme forme ouverte à modeler. On peut retenir de cette ouverture la perte de sens qui en découle et l'angoisse que cette perte peut susciter. On peut également souligner le potentiel de liberté que cette ouverture insuffle sur l'espace social, sur le réel référentiel et au-delà de celui-ci. (Semprini, 2003) 


\section{Bibliographie}

BESSON, Anne (2006). 'Le mythe culturel en fiction: deux relectures de la préhistoire arthurienne par la fantasy contemporaine". In: Durand-Le Guern, (dir.). Images du Moyen Age. PU de Rennes "Interférences", pp 175-184.

BESSON, Anne (2007). "Une histoire infinie...quelques pistes d'explication théorique pour la pérennité d'Arthur". In: Anne Besson (ed), Le roi Arthur au miroir du temps. Dinan: Terre de Brume.

BESSON, Anne (ed.) (2007). Le roi Arthur au miroir du temps. Dinan: Terre de Brume.

DuRAND-Le GueRn (dir.) (2006). Images du Moyen Age. PU de Rennes "Interférences".

CASETTI CASETTI, Francesco, ODIN, Roger (1990). "De la paléo- à la néo-télévision ». In: Communications, no 51, pp 9-26.

ChAmBAT, Pierre, EHREnBERG, Alain (1993). "Les reality shows, un nouvel âge télévisuel ?". In: Esprit, Janvier, pp 5-13.

Eco, Umberto (1985). La guerre du faux. Paris: Grasset.

MEHL, Dominique (2002). "La télévision relationnelle". In: Cahiers internationaux de sociologie, 1, n 112, pp 63-95.

MercieR, André, PelletieR, Esther (dir (s)) (1999). L'adaptation dans tous ses états. Québec: Nota Bene.

MISSIKA, Jean Louis (2006). La fin de la télévision. Paris: Seuil.

SAINT-Gelais, Richard (1999). "Adaptation et transfictionnalité". In: Mercier, André, Pelletier, Esther (dir (s)). L'adaptation dans tous ses états. Québec: Nota Bene.

SEMPRINI, Andréa (2003). La société des flux. Formes du sens et identité dans les sociétés contemporaines. Paris: L'Harmattan. 